\title{
Research on real time telemetry system in bicycling training Hongbing $\mathrm{Ba}^{1, a}$, Jihe Zhou ${ }^{2, b}$ \\ ${ }^{1}$ Department of Physical Education, Aba Teachers University, Aba 623002, China \\ ${ }^{2}$ Department of Sports Medicine, Chengdu Sport University, Chengdu, 610041, China \\ aemail: bahongbing.2007@163.com, bemail: jihezhou123@163.com
}

Keywords: Bicycling training; Long distance training; Real time telemetry system

\begin{abstract}
In this paper, the GPS global positioning system, Polar wearlink and imaging technology, the combination of a set of software and hardware system, applied to the long distance bicycling training, it aims to get the information in training, including the video track, speed, heart rate and other parameters. By the combination of real time data and post processing so as to develop bicycling training principles and contribute to sports science innovation.
\end{abstract}

\section{Introduction}

Bicycling began from the first session of the modern Olympic Games. It is very popular sport in Europe and United States, both commercial and professional development are very well in these countries. The best known "Tour of France" is the third sports event right after football World Cup, which reflects its popularity and influence in the world [1].

The main objective of this paper is study movement trail in long distance exercise, heart rate, speed, riding posture and real-time telemetry. Use the telemetry data to make coaches master the real time training states of athletes, in addition to the analysis of the training data processing, to improve the scientific bicycling training.

\section{Research methods}

Design of real time telemetry system derives from the training needs, and also the need of development of modern science and technology. The design of the GPS global positioning system, Polar belt and camera technology combined of a set of software and hardware system gets real time data in the long distance cycling training, including the video track, speed, heart rate and other parameters.

The working mechanism of real time telemetry system is use GPS and Polar wearlink to record player's information and transmit the information to the receiver at the real time; use the camera records the athletes riding posture, summarizes information on a laptop for display and storage, to achieve real-time remote telemetry (Figure 1). GPS and Polar wearlink has its real time method to achieve data synchronization. After the data transmission, the specialized software on a laptop can store data and analyze the situation of training through the stored heart rate and other information. The analysis report can play a good supporting role in scientific bicycling training.

\section{Results and discussion}

\section{The main technical route of real-time telemetry system.}

The advantages of GPS system: global, all-weather work; high precision; multiple functions, wide application. And its real time telemetry can be applied in bicycling training. The GPS system in this experiment adapts GPSports SPI10 GPS made in Australia, the main technical parameters of GPS:

- 12 parallel channels (at most 12 satellite positioning)

- Position: hot start time $<15$ seconds $<45$ seconds; cold start; To capture $<2$ seconds; automatic search for $<300$ seconds

- New rate: 5 times / sec 
- Speed: accuracy of better than $0.1 \mathrm{M} / \mathrm{s}$

- The positioning accuracy is better than 15 meters

- Speed limit: >500 M / s

- Acceleration limit: $6 \mathrm{~g}$

- Ambient temperature: (1) the working temperature: -30 degrees to +80 degrees $C$; (2) the storage temperature: -40 degrees to +80 degrees $C$

Polar belt is a widely used instrument in athletes' heart rate telemetry can collect the beating of heart shock, transform data into electrical signals and transmit to the collector. The study on the heart rate zone makes the necessary changes in heart rate, directly bring additional heart rate data memory and it will be used the data line is connected with the transmitter.

The GPS data transmitter and heart rate with the collected through electromagnetic waves (in the range of harmless to the human body) sent to the receiver. The transmitter is in the form of a semicircle shape cell wave transmitting, the radius can reach 1000 meters, which can facilitate receiving data in a long distance from the player's position.

Laptop with receiver, usually connected to a video camera, remote control system for the working mode, real time processing (GPS data and video and telemetry) analysis and post processing system.

Post processing system includes: GPS and heart rate data processing system and video processing system.

GPS and heart rate of the data processing using specially designed software GPSVideoV1.0CKey. For data analysis, can be the heart rate, speed, video and other data recorded by charts and curves reflect the form; can be integrated into the trajectory of the latitude and longitude, and can find a point of the heart the data in the track, speed, can also be synchronized video query, so that the heart rate, speed, trajectory, video display on the same screen.

Video processing software is developed by Visual Basic 6 computer programming language, this software provides the angle, length / distance, angular velocity, average velocity, angular velocity and average velocity kinematics data through the technique of video analysis, promising a quantitative data reference for bicycling training.

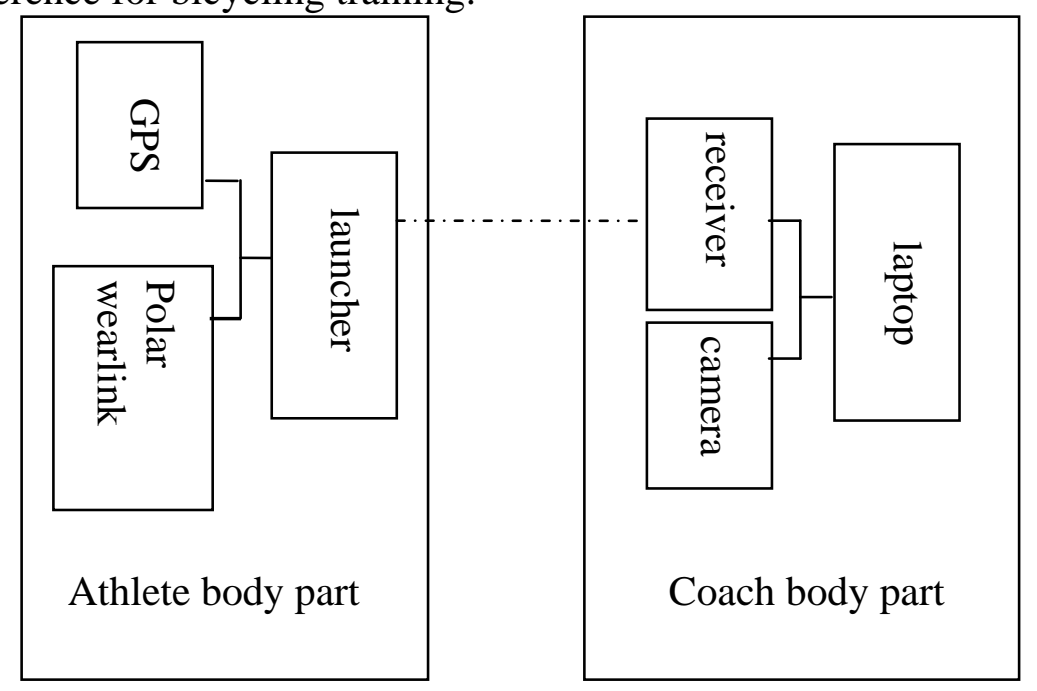

Figure 1 Schematic diagram of real-time telemetry system

\section{Real time telemetry data and a simple analysis of the training of athletes.}

1)Athletes heart rate telemetry

Heart rate was one of physiological indexes often used in sports training, it is a very sensitive physiological index to reflect the metabolic rate, the work of heart rate monitoring in the process of sports training is very serious. According to the relationship between the changes of exercise load and body function, heart rate and exercise intensity, photo there are significant linear relationship between oxygen and energy metabolism in exercise, until the time of maximum load in the load 
movement, along with the gradual increase in intensity, energy metabolism and oxygen demand more and more, the heart rate index will become much higher than normal. Through the experiments of real-time dynamic change monitor athletes heart rate, heart rate information in real time of exercise, the body reacts by radio waves transmitted in real time to the receiver, can make the coaches easily manage the intense exercise; through the analysis of the heart rate curve in the process of training in practice can be observed in the training characteristic of bicycle training after the training, system can analyze the state, mental state of athletes, but also the process and results of the competition of scientific analysis, in order to achieve the ideal effect, which is also used in this experiment[2].

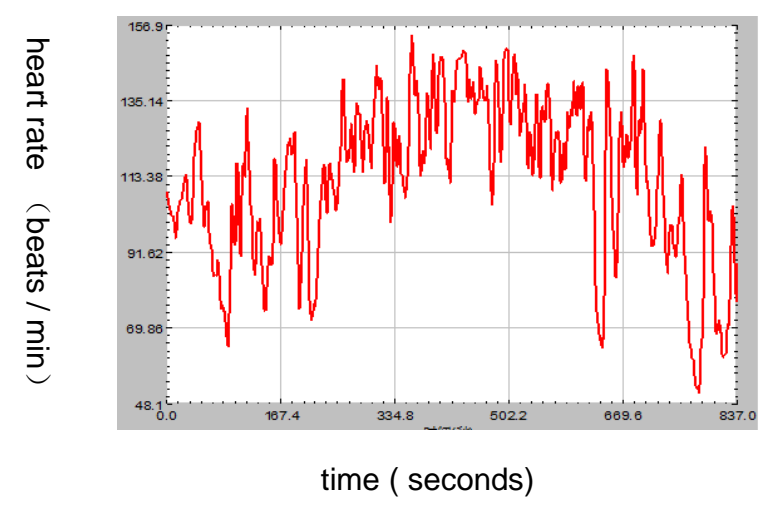

Figure 2 Heart rate of middle and long distance bicycle exercise training

Figure 2 is the heart rate in the experimental process of athlete, we can clearly observe the athletes' heart rate in training. At beginning of the chart, athlete's heart rate is very high in upward trend. This is because the body is in motion, muscle strength, intensify energy consumption, the body of oxygen demand increased, cardiovascular activity strengthened, so that the heart rate. As the movement, athlete is used to the movement, break though physical limit, heart rate remained relatively stable state in their respective.

2) Athletes rate telemetry

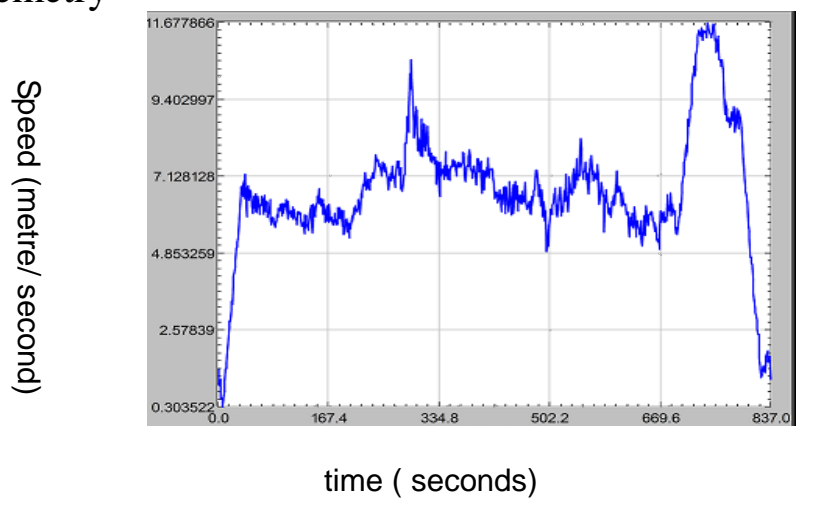

Figure 3 Long distance bicycle speed telemetry in sports training

From Figure 3 can clearly read telemetry instant rate, conducive to the coaches of the telemetry and scientific guidance. In the actual training, due to the long distance riding is a typical endurance sports, training process is very hard, and in this case, coaches can depend on real-time telemetry, the biological basis of heart rate based on the knowledge of some athletes in the training process, judge the deceleration the real fatigue, or the negative training athletes. Timely regulate the amount of training or urge the athletes training, so as to ensure the quality of training, scientific training.

3) Synchronous display

Synchronous display includes data and video synchronization display plate and map based data processing of the plate. Plate mainly used to analyze the data curve of arbitrary values in a gesture of the athletes riding data and video display synchronization; video synchronous display and map based data display plate is mainly used to show the trajectory of an arbitrary point in speed, heart rate and the synchronization of the riding posture, kinematics analysis and the interface of athletes 
riding posture further quantitative 2D.

Through the training after the treatment of the synchronous remote sensing images through video analysis and get the angle data characteristics for coaches to monitor players' exercise, so as to provide quantitative data reference for bicycle exercise training, the training method and the experience of sports coaches tend to have specific qualitative quantitative control scientific training methods, in order to change the present situation of exercise training, find out the main reason of insufficient exercise . Data and video synchronization display plate is the key part of post processing.

\section{Conclusions}

Real time telemetry system has the advantages of light weight, reliable data, no side effect to the athlete's normal training etc. It can get the heart rate, speed of athletes in the training process and different riding posture in real time, the post-processing can display the heart rate, speed, trajectory and videos, which reflects the processing capacity and deformation curve of athletes with physical decline and whether the technical movements changed, with the latter part of the video analysis of the angle data, in order to facilitate the coaches scientific guidance to guarantee the quality of training, training methods and the experience of coaches athletes feeling, to control with scientific training methods, so as to improve the athlete's speed ability.

\section{Acknowledgements}

In this paper, the research was sponsored by the Sichuan provincial department of science and technology (Project No. 2008SZ0219).

\section{References}

[1] wei li.Thoughts on the current situation and training Chinese bicycle exercise science[J]. Journal of Harbin Institute Of Physical Education.2006.

[2]wenjun Wan. The change of heart rate and scientific design, monitoring and evaluation of physical exercise [J]. Journal of Guangzhou Sports University, 2003, 3 (23). 